\title{
TP75-MicroCT Test Bed Data Acquisition for Multi-Energy Zeff and Rho-e Decomposition Analysis
}

\author{
William D. Brown and Jerel A. Smith \\ Lawrence Livermore National Laboratory \\ Livermore, CA 94551 \\ Work performed on the \\ Science \& Technology Directorate of the \\ Department of Homeland Security \\ Statement of Work \\ PR RSEN-08-00066
}

January 23, 2013

Version 3.0

LLNL-TR-615552
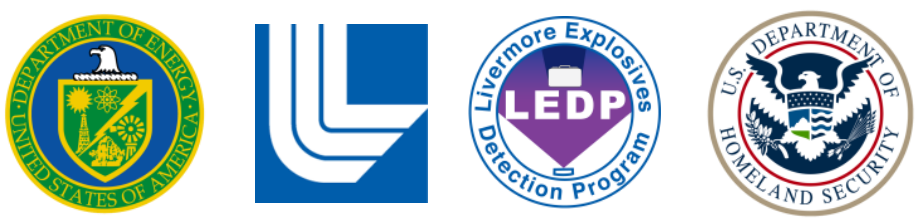
This document was prepared as an account of work sponsored by an agency of the United States government. Neither the United States government nor Lawrence Livermore National Security, LLC, nor any of their employees makes any warranty, expressed or implied, or assumes any legal liability or responsibility for the accuracy, completeness, or usefulness of any information, apparatus, product, or process disclosed, or represents that its use would not infringe privately owned rights. Reference herein to any specific commercial product, process, or service by trade name, trademark, manufacturer, or otherwise does not necessarily constitute or imply its endorsement, recommendation, or favoring by the United States government or Lawrence Livermore National Security, LLC. The views and opinions of authors expressed herein do not necessarily state or reflect those of the United States government or Lawrence Livermore National Security, LLC, and shall not be used for advertising or product endorsement purposes.

This work performed under the auspices of the U.S. Department of Energy by Lawrence Livermore National Laboratory under Contract DE-AC52-07NA27344. 


\title{
TP75-MicroCT Test Bed and HEAF Data Acquisition for Multi-Energy Zeff and Rho-e Decomposition Analysis
}

\author{
William D. Brown and Jerel A. Smith \\ Lawrence Livermore National Laboratory \\ Livermore, CA 94551
}

\section{Objectives}

LLNL has been tasked to develop a system independent method that will characterize Home Made Explosives (HME) materials. The objective of this test plan is to acquire Computed Tomography (CT) data on two LLNL systems (MCT-TB and HEAF). The CT data will be used in evaluating potential System Independent Decomposition methods.

\section{Summary of Approach}

LLNL will acquire MicroCT tomography data at different spectral energies using two (MCT-TB and HEAF) systems. The data acquired will include known references that are housed in the lower tray of the MicroCT carousel and known samples located in the upper tray of the MicroCT carousel. The same references and samples will be used for both systems.

\section{Materials and Specimens:}

a) MicroCT Carousel with references [Smith1]

b) Test Materials (see Table 1 and Figure 1)

A total of 7 specimens will be scanned in each spectral configuration. Specimens 1-4 are simple solid right-circular cylindrical objects. Specimens 5 (Teflon Plug) and 6 (Delrin Plug) are assembled from multiple components as shown in Figure 1. Specimen 7 is water in the standard 60-ml bottle.

The Teflon and Delrin plugs contain 4 cylindrical cutouts. The cutouts are used to house the 10-mm Samples and water. Figure 1 contains a schematic (top view) showing the assembled Specimens 5 and 6 including the plugs with the corresponding sample inserts and their locations. 


\begin{tabular}{|c|c|c|c|c|c|}
\hline Name & Reference & LEDP ID & Temp ID & Dia (mm) & Length $(\mathrm{mm})$ \\
\hline Specimen 1 & Graphite & LR-GRA-02-02 & A6 & 50.8 & 25.4 \\
\hline Specimen 2 & Teflon & LR-TEF-01-01 & A7 & 56 & 50.8 \\
\hline Specimen 3 & Magnesium & LR-MAG-01-02 & $\mathrm{A} 8$ & 25.4 & 50.8 \\
\hline Specimen 4 & Silicon $^{1}$ & LR-SIL-01-02 & A9 & 25.4 & 25.4 \\
\hline Insert A & Teflon & LR-TEF-01-03 & A13 & 10 & 50.8 \\
\hline Insert B & Delrin & LR-DEL-02-03 & A12 & 10 & 50.8 \\
\hline Insert C & Magnesium & LR-MAG-01-03 & A14 & 10 & 50.8 \\
\hline Insert D & Water $^{2}$ & N/A & N/A & N/A & N/A \\
\hline Specimen 5 & Teflon Plug & LR-TEF-01-02 & A10 & 56 & 25.4 \\
\hline Specimen 6 & Delrin Plug & LR-DEL-02-03 & A11 & 50.8 & 25.4 \\
\hline \begin{tabular}{|l|} 
Specimen 7 \\
\end{tabular} & Water $^{2}(60 \mathrm{ml})$ & N/A & $\mathrm{N} / \mathrm{A}$ & $36.9 / 38.9$ & $\sim 55$ \\
\hline
\end{tabular}

${ }^{1}$ Operator will wear Nitrile Gloves while handling the Silicon sample

${ }^{1}$ Deionized Water, Fisher Scientific, Cat \# 23-751-610,

http://www.fishersci.com/ecomm/servlet/fsproductdetail ? catalogId=29103\&productId=1 $1963016 \&$ langId=-1\&store $I d=10652 \&$ distype $=2 \&$ isChemical=false $\&$ fromSearch $=0$

Table 1. Materials to be used for data acquisition, either as bare specimens (1-4) or as components in the assembled Specimens 5 and 6.

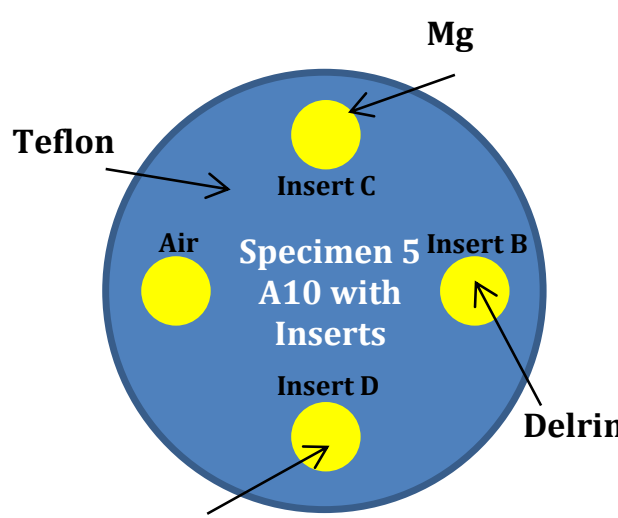

Water

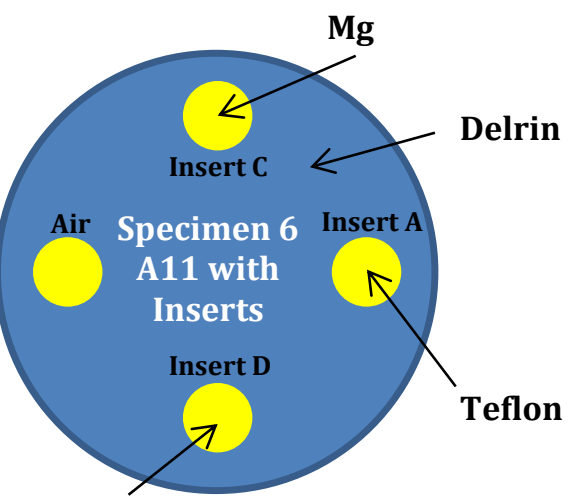

Water

Figure 1. Schematic showing the Teflon (Specimen 5) and Delrin (Specimen 6) with inserts and insert locations (top view)

\section{X-ray Systems:}

a) MCT-TB and HEAF MicroCT Systems [Smith] 


\section{System Spectra}

The CT data will be acquired at five separate X-ray spectra for the MCT-TB system and two separate spectra for the HEAF system. Table 2 defines the spectrum, filtering and which system will be used for each spectrum.

\begin{tabular}{|c|c|c|c|c|}
\hline Name & $\mathbf{k V p}$ & Filter & System & Detector \\
\hline Spectrum 1 & 100 & $2 \mathrm{~mm} \mathrm{Al}$ & HEAF & Thales \\
\hline Spectrum 1A & 100 & $1.25 \mathrm{~mm} \mathrm{Al}$ & MCT-TB & Perkin Elmer \\
\hline Spectrum 2 & 160 & $2 \mathrm{~mm} \mathrm{Al} \mathrm{\&} \mathrm{2mm} \mathrm{Cu}$ & HEAF & Thales \\
\hline Spectrum 2A & 160 & $1.25 \mathrm{~mm} \mathrm{Al} \mathrm{\&} \mathrm{2mm} \mathrm{Cu}$ & MCT-TB & Perkin Elmer \\
\hline Spectrum 3 & 80 & $0.5 \mathrm{~mm} \mathrm{Al}$ & MCT-TB & Perkin Elmer \\
\hline Spectrum 4 & 125 & $1 \mathrm{~mm} \mathrm{Cu}$ & MCT-TB & Perkin Elmer \\
\hline Spectrum 5 & 200 & $3 \mathrm{~mm} \mathrm{Cu}$ & MCT-TB & Perkin Elmer \\
\hline
\end{tabular}

Table 2. $\mathrm{kVp}$ and tube-head filtering requirements for each spectrum

\section{CT Technique Parameters}

For data acquired at each spectrum and for each system, a technique sheet will be prepared that outlines the CT technique parameters. The parameter spreadsheet is located in Appendix A and the Excel workbook can be found under:

$\boldsymbol{W}: \backslash$ Documents\Documents $\backslash$ Testplans\TP75_MCT_TB_Multi_Energy_Decomposition_Pl an $\backslash$ Spreadsheets $\backslash$ Technique_Template.xlsx

Where:

W: Is the Windows ${ }^{\circledR}$ drive letter associated with the LEDP Working Directory

All MicroCT technique sheets will be placed on the LEDP server. All directory paths and filenames should NOT contain any spaces. Use an underscore " " symbol for a space. The data shall be filed as follows:

W:\Working $\backslash T P 75 \_M C T \_T B \_M u l t i \_E n e r g y \_D e c o m p o s i t i o n \backslash L L N L W o n e \backslash T e c h n i q u e s \backslash S p e$ ctra_Num_System

Where:

W: - Is the Windows ${ }^{\circledR}$ drive letter associated with the LEDP Working Directory

Num - is the spectrum number 1, 1A, 2, 2A, 3, 4 or 5

System - is the HEAF or MCTTB

For the spectra listed in section 5, tube current and detector integration times may need to be determined. The procedure below outlines the process to acquire tube current and detector integration times for each system at each defined spectrum.

Spectra 1, 1A, 2 and 2A:

- For both the MCT-TB and HEAF systems, the tube current and integration times (knob selection for HEAF) have been determined. 
Spectra 3, 4 and 5 (MCT-TB Only):

- For each energy $(\mathrm{kVp})$ selection, perform the following:

- Select the maximum tube current that can be achieved with the $0.4 \mathrm{~mm}$ spot

- Place the slit collimator in front of the detector

- Set integration time to 0.3 seconds

- Acquire Raw image

- Plot a lineout through the top slit to obtain detector counts

- If the maximum detector counts are $45000+/-3000$, then the integration time will be used for the technique. If the counts are less than $45000+/-3000$ the integration will be increased. If the counts are greater than $45000+/-3000$ the integration time will be reduced. Continue changing the integration times until an acceptable integration times has been determined.

- Add the final tube current and integration times to the technique sheets

\section{System Alignment}

The MicroCT system must be aligned before any data acquisition. If the system is not aligned or there is reason to believe it may be out of alignment, the system will be aligned using the fine alignment SOP[Smith2]

\section{Acquiring Calibration Files}

Detector panel calibration files will be acquired once a week. The calibration files (Dark, Light and Mid) will be acquired at each spectrum using the corresponding spectrum filtration and integration times (knob selection for the HEAF). The calibration files will be used to calibrate the panel for all data acquisitions until a new set is acquired. To acquire calibration files follow the procedure outlined below:

8.1 Select the proper $\mathrm{kVp}$, filtration and integration times ( $\mathrm{knob}$ selection for HEAF) for Spectrum 1A (MCT_TB) or Spectrum 1 (HEAF).

8.2 Remove the copper strip from the detector and remove the Slit Collimator from the field of view

8.3 Turn on the x-rays and wait 2 minutes

8.4 Run the calibration mode from the data acquisition software and acquire an image. Use 16 frame averages.

8.5 Adjust the tube current until the image contains a maximum of $45000+/$ 3000 counts. This is the Light calibration file.

8.6 Adjust the tube current to $65 \%$ of the Light field current. Acquire another image using 16 frame averages. This is the Mid calibration file.

8.7 Turn off $x$-rays and wait 2 minutes.

8.8 Acquire another image with $\mathrm{x}$-rays off with 16 frame average. This is the Dark calibration filed.

8.9 Save the calibration files with unique filenames and inside the xtech directory

8.10 Repeat steps 8.1 thru 8.9 for the other spectra 


\section{HEAF MicroCT Procedure}

The HEAF MicroCT procedure uses only Spectrum 1 and Spectrum 2. CT parameters shall be setup before data acquisition using the corresponding CT Technique Sheet (see section 6).

9.1 Acquire CT parameters from the CT Technique sheet for Spectrum 2

9.2 Place Specimen 1 in the carousel sample position using the corresponding carousel puck.

9.3 Remove the Copper strip from the panel

9.4 Place the slit collimator into position

9.5 Set Thales Panel integration knob to the correct setting (see technique sheet)

9.6 Place the correct tube filtration (see technique sheet) at the tube head port.

9.7 Select the correct $\mathrm{kVp}$ and Tube Current (see technique sheet) on the $\mathrm{x}$-ray controller

9.8 Move carousel out of the field of view

9.9 Turn on X-rays and wait 2 minutes

9.10 Acquire pre-CT I0 Image with 16 frame averaging

9.11 Turn off x-rays, place carousel in field of view, place copper strip on the detector.

9.12 Turn on x-rays and wait 2 minutes

9.13 Acquire CT data per Spectrum 2 Technique sheet

9.14 Turn off x-rays and remove copper strip from the detector and move carousel out-of-view.

9.15 Turn on $\mathrm{x}$-rays and wait 2 minutes

9.16 Acquire post-CT I0 Image with 16 frame averaging

9.17 Repeat steps 9.1 thru 9.16 using Spectrum 1

9.18 Repeat Steps 9.1 through 9.17 for Specimens 2, 3, 4, 5 6, and 7. NOTE: For Specimens 5 and 6, the water sample in the cylindrical plug will be placed such that it is closest to the $\mathrm{x}$-ray source.

\section{MCT-TB Procedure}

The MCT-TB procedure uses two sets of spectra.

Spectral Set 1: Spectrum 2A and Spectrum 1A. These spectra should be run in that order, high energy before low energy.

Spectral Set 2: Spectrum 5, Spectrum 4, and Spectrum 3. These spectra should be run in that order, high energy before low energy.

Spectral Set 1 and Spectral Set 2 must each be run in a single day, and with minimal delay between the separate spectra. Do not touch or move the specimen 
between spectra or between spectral sets. It is not important that Spectral Set 1 be run before Spectral Set 2.

The MCT-TB_1 procedure uses Spectra 2A, and 1A. CT parameters shall be setup before data acquisition using the corresponding CT Technique Sheet (see section 6).

10.1 Acquire CT parameters from the CT Technique sheet for Spectrum 2A

10.2 Place Sample 1 in the carousel sample position using the corresponding carousel puck.

10.3 Remove the Copper strip from the panel

10.4 Place the slit collimator into position

10.5 Place the correct tube filtration (see technique sheet) at the tube head port.

10.6 Select the correct $\mathrm{kVp}$ and Tube Current (see technique sheet) on the $\mathrm{x}$-ray controller

10.7 Move carousel out of the field of view

10.8 Turn on $\mathrm{x}$-rays and wait 2 minutes

10.9 Select the correct integration time (see technique sheet). Acquire pre-CT I0 Image with 16 frame averaging

10.10 Turn off x-rays, place carousel in field of view, place copper strip on the detector.

10.11 Turn on $\mathrm{x}$-rays and wait 2 minutes

10.12 Acquire CT data per Spectrum 2A Technique sheet

10.13 Turn off x-rays and remove copper strip from the detector, and move carousel out of field of view

10.14 Turn on $\mathrm{X}$-rays and wait 2 minutes

10.15 Acquire post-CT I0 Image with 16 frame averaging

10.16 Repeat steps 10.1 thru 10.15 using Spectrum 2A

10.17 Repeat Steps 10.1 through 10.16 for Specimens 2, 3, 4, 5, 6 and 7.

NOTE: For Specimens 5 and 6, the water sample in the cylindrical plug will be placed such that it is closest to the $\mathrm{x}$-ray source.

The MCT-TB_2 procedure uses Spectra 5, 4, and 3. CT parameters shall be setup before data acquisition using the corresponding CT Technique Sheet (see section 6).

10.18 Acquire CT parameters from the CT Technique sheet for Spectrum 5

10.19 Place Sample 1 in the carousel sample position using the corresponding carousel puck.

10.20 Remove the Copper strip from the panel

10.21 Place the slit collimator into position

10.22 Place the correct tube filtration (see technique sheet) at the tube head port. 
10.23 Select the correct $\mathrm{kVp}$ and Tube Current (see technique sheet) on the $\mathrm{x}$-ray controller

10.24 Move carousel out of the field of view

10.25 Turn on $\mathrm{x}$-rays and wait 2 minutes

10.26 Select the correct integration time (see technique sheet). Acquire pre-CT I0 Image with 16 frame averaging

10.27 Turn off $x$-rays, place carousel in field of view, place copper strip on the detector.

10.28 Turn on $\mathrm{x}$-rays and wait 2 minutes

10.29 Acquire CT data per Spectrum 5 Technique sheet

10.30 Turn off $\mathrm{x}$-rays and remove copper strip from the detector, and move carousel out of field of view

10.31 Turn on $\mathrm{x}$-rays and wait 2 minutes

10.32 Acquire post-CT I0 Image with 16 frame averaging

10.33 Repeat steps 10.18 thru 10.32 using Spectrum 4

10.34 Repeat steps 10.18 thru 10.32 using Spectrum 3

10.35 Repeat Steps 10.18 through 10.34 for Specimens 2, 3, 4, 5, 6 and 7.

NOTE: For Specimens 5 and 6, the water sample in the cylindrical plug will be placed such that it is closest to the x-ray source.

\section{Photographs}

Photographs of each test sample will be acquired. All MicroCT photographs will be placed on the LEDP server. All directory paths and filenames should NOT contain any spaces. Use an underscore "_" symbol for a space. The data shall be filed as follows:

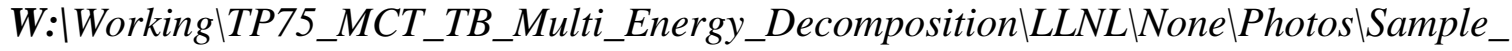
Num

Where:

W: - Is the Windows ${ }^{\circledR}$ drive letter associated with the LEDP Working Directory A photograph of each system showing the $\mathrm{x}$-ray source, staging, carousel and detector will be acquired. The data shall be filed as follows:

W:\Working \TP75_MCT_TB_Multi_Energy_Decomposition $\backslash L L N L W o n e \backslash P h o t o s \backslash$ System Where:

W: - Is the Windows ${ }^{\circledR}$ drive letter associated with the LEDP Working Directory System - Is the MicroCT system (MCTTB or HEAF) 


\section{Data Transfer}

All MicroCT data will be placed on the LEDP server. All directory paths and filenames should NOT contain any spaces. Use an underscore "_" symbol for a space. The data shall be filed as follows:

W:\Working $\backslash T P 75 \_M C T \_T B \_M u l t i \_E n e r g y \_D e c o m p o s i t i o n \backslash L L N L W o n e \backslash S y s t e m \backslash$ None $\backslash Y$ YMMDD_Spectra_SPNum_Sample_SampNum

Where:

W: - Is the Windows ${ }^{\circledR}$ drive letter associated with the LEDP Working Directory

YYMMDD - Is the acquisition date

System - is the HEAF or MCTTB

SPNum - is the spectrum number 1, 2, 3, 4 or 5

SampNum - is the specimen number 1, 2, 3, 4, 5 and 6.

\section{References}

Smith1 Smith, Jerel A. and Daniel Schneberk, Jeffrey Kallman, Harry Martz, "Documentation of the LLNL and Tyndall MicroComputed Tomography System", Lawrence Livermore National Laboratory, 17 December, 2009

Smith2 Fine alignment SOP 


\section{Appendix A - Technique Sheet Template}

\begin{tabular}{|l|l|}
\hline Title & \\
\hline System Information & \\
\hline Data Acquistion Date & \\
\hline System & \\
\hline Archive Directory & \\
\hline Source & \\
\hline X-ray Source & Yxlon 450kV D09 \\
\hline Effective Spot Size (mm) & Small 0.4 \\
\hline Energy (kV) & \\
\hline Tube Curren t (mA) & \\
\hline Unsharpness (mm) & \\
\hline Filter (Type/Thickness mm) & \\
\hline & \\
\hline Detector & \\
\hline Detector Type & Amorphous Silicon Thales/Perkin Elmer \\
\hline Source-Detector-Distance (SDD) (mm) & \\
\hline Source-Object-Distance (SOD) (mm) & \\
\hline Object-Detector-Distance (ODD) (mm) & \\
\hline X-Offset (Pixels) & \\
\hline X-Size (Pixels) & \\
\hline Y-Offset (Pixels) & \\
\hline Y-Size (Pixels) & \\
\hline Magnification & \\
\hline Frame Average(s) & \\
\hline Integration Knob-Thales Only & \\
\hline Integration Time per Projection(sec) & \\
\hline Raw Pixel Size (mm) & \\
\hline Global Resampling & \\
\hline Effective Pixel size at Detector (mm) & \\
\hline Effective Pixel size at Object (mm) & \\
\hline & \\
\hline CT Parameters & \\
\hline Numbers of Views & \\
\hline Number of Angles & \\
\hline Delta Angle (Degrees) & \\
\hline Estimated PxCenter (Pixel) & \\
\hline Estimated PzCenter (Pixel) & \\
\hline & \\
\hline
\end{tabular}

